\title{
Impaired angiogenic potency of bone marrow cells from patients with advanced age, anemia, and renal failure
}

Tao-Sheng Li, MD, PhD, Masayuki Kubo, PhD, Kazuhiro Ueda, MD, PhD, Masanori Murakami, MD, PhD, Akihito Mikamo, MD, PhD, and Kimikazu Hamano, MD, PhD

Objective: The implantation of autologous bone marrow-derived cells has been used for the treatment of ischemic diseases, but obvious interindividual differences were observed in the improvement of regional perfusion and cardiac function after treatment. We examined the angiogenic potency of bone marrow cells from patients with different clinical backgrounds.

Methods: Bone marrow cells were collected from 25 patients scheduled to undergo sternotomy for various surgical procedures. We examined the quality of bone marrow cells and investigated their angiogenic potency by using an ischemic limb model in mice with severe combined immunodeficiency.

Results: When compared with their control cohort, bone marrow cells from patients with advanced age, renal failure, or anemia had significantly less c-kit- and CD34-positive stem cells $(P<.05)$ and showed significantly lower vascular endothelial growth factor production and colony-forming units in culture $(P<.05)$. Furthermore, the implantation of bone marrow cells from patients with advanced age, renal failure, or anemia into the ischemic limbs of mice also resulted in significantly worse blood flow recovery and clinical score when compared with the implantation of bone marrow cells from their control cohorts $(P<.05)$. However, the bone marrow cells from patients with diabetes and hypertension did not show significant impairment of angiogenic potency when compared with their control cohorts.

Conclusions: The quality and angiogenic potency of bone marrow cells differs among patients. Advanced age, renal failure, and anemia should be the risk factors related to poor angiogenic potency of bone marrow cells for the treatment of ischemic diseases. (J Thorac Cardiovasc Surg 2010;139:459-65)

Bone marrow-derived (stem) cells have been used to induce angiogenesis for the treatment of ischemic diseases, ${ }^{1-4}$ and the clinical application of autologous cells has no ethical or immunologic problems. However, randomized controlled trials have reported conflicting data about the beneficial effects of using autologous bone marrow cells for the treatment of ischemic cardiovascular diseases. ${ }^{2-4}$ Furthermore, obvious interindividual differences in the improvement of regional perfusion and cardiac function were observed after the implantation of autologous bone marrow cells. ${ }^{1-4}$ A subgroup analysis of the REPAIR-AMI study revealed that a good outcome was observed in patients with large infarct size and poor left ventricular function. ${ }^{4}$ Conversely, the BOOST study suggested that the outcomes of bone marrow cell implantation were not related to the patient's age, left ventricular function,

From the Department of Surgery and Clinical Science, Yamaguchi University Graduate School of Medicine, Yamaguchi, Japan.

Disclosures: None.

This work was supported by a Grant-in-Aid for Scientific Research from the Ministry of Education, Science, Sports, and Culture.

Received for publication Jan 13, 2009; revisions received July 1, 2009; accepted for publication July 22, 2009; available ahead of print Sept 14, 2009.

Address for reprints: Tao-Sheng Li, MD, PhD, Department of Surgery and Clinical Science, Yamaguchi University Graduate School of Medicine, 1-1-1 Minami-

Kogushi, Ube, Yamaguchi, Japan 755-8505 (E-mail: litaoshe@yamaguchi-u.ac.jp). 0022-5223/\$36.00

Copyright $(c) 2010$ by The American Association for Thoracic Surgery

doi:10.1016/j.jtcvs.2009.07.053 or time to cell delivery. ${ }^{2}$ However, we do not exactly know the factor that influences the outcome of therapy.

The mechanism of the induction of therapeutic angiogenesis by bone marrow cells is extremely complex, involving the quality of the cells used for implantation and the condition of the targeted ischemic tissue. ${ }^{5,6}$ Many factors, such as aging, diabetes, and other systemic diseases have been found to contribute to the functional impairment of bone marrow cells. ${ }^{7-10}$ This might explain poor outcomes in some patients after autologous bone marrow cell implantation for the treatment of ischemic heart diseases. ${ }^{1-4}$ Therefore it is critically important to identify the risk factors that contribute to the functional impairment of bone marrow cells for inducing therapeutic angiogenesis.

In this study we collected bone marrow cells from patients with different clinical backgrounds. We examined the quality of bone marrow cells by means of in vitro assessments and also investigated their angiogenic potency using an ischemic limb model in mice with severe combined immunodeficiency (SCID mice). We found that advanced age, renal failure, and anemia were the risk factors that contributed to poor angiogenic potency of the bone marrow cells of patients.

\section{MATERIALS AND METHODS \\ Collection of Bone Marrow Cells From Patients With Different Clinical Backgrounds \\ Twenty-five patients (age range, approximately $44-84$ years; mean age, $65.1 \pm 10.7$ years) were enrolled in this study. The diagnoses of these}




\section{Abbreviations and Acronyms \\ SCID mice $=$ mice with severe combined immunodeficiency \\ VEGF $=$ vascular endothelial growth factor}

patients were ischemic heart disease in 10 patients, valve disease in $12 \mathrm{pa}$ tients, thymoma in 2 patients, and patent ductus arteriosus in 1 patient. All patients underwent a scheduled operation performed through a sternotomy. The ethics review board at our university approved this study, and written informed consent was obtained preoperatively from every patient.

About $15 \mathrm{~mL}$ of bone marrow fluid was aspirated from the sternum before sternotomy for surgical procedure. Bone marrow mononuclear cells were isolated by means of density gradient centrifugation. An average of $1.35(1.35 \pm 1.12) \times 10^{8}$ bone marrow mononuclear cells were collected from 1 patient, and freshly isolated cells were used immediately for experiments.

\section{Characterization of Bone Marrow Cells}

The expression of CD34 and c-kit in freshly isolated bone marrow cells was measured by means of flow cytometry, as described previously. ${ }^{11}$ Briefly, cells were incubated for 30 minutes at $4{ }^{\circ} \mathrm{C}$ with fluorescein isothiocyanate-conjugated mouse anti-human CD34 antibody and phycoerythrinconjugated mouse anti-human c-kit antibody (Miltenyi Biotec, Bergisch Gladbach, Germany). Quantitative flow cytometric analysis was done with a FACS SCAN flow cytometer and Cell Quest software (Becton Dickinson, Franklin Lakes, NJ).

\section{Evaluation of the Potency of Vascular Endothelial Growth Factor Production and Colony Formation of Bone Marrow Cells}

Vascular endothelial growth factor (VEGF) production and colony formation from bone marrow cells was evaluated as described previously. ${ }^{8}$ Briefly, freshly isolated bone marrow mononuclear cells from each patient were suspended in RPMI 1640 medium supplemented with $10 \%$ fetal bovine serum (Gibco BRL, Carlsbad, Calif) and cultured at $37^{\circ} \mathrm{C}$ in a humidified environment with $5 \% \mathrm{CO}_{2}$. After 3 days of culture on 96 -well plates, supernatant was collected, and the concentration of VEGF in the supernatant was measured with ELISA kits (R\&D Systems, Minneapolis, Minn). Colony-forming assay was done by culturing cells on a BIOCOAT CELLWARE 24-well plate (Becton Dickson Labware, Bedford, Mass), and the number of colony-forming units was counted after 10 days of cultivation. ${ }^{8}$

\section{Ischemic Hind-Limb Model of Mice and the Implantation of Patient's Bone Marrow Cells}

We investigated the angiogenic potency of the patient's bone marrow cells in vivo by using an acute ischemic hind-limb model in SCID mice. ${ }^{12}$ Briefly, an ischemic hind-limb model was created in 12- to 15week-old mice. After the initiation of limb ischemia, bone marrow mononuclear cells from patients were injected intramuscularly, with a total of $1 \times 10^{6}$ cells at 4 points (each with $2.5 \times 10^{5}$ cells $/ 10 \mu \mathrm{L}$ of saline) in the ischemic hind limbs of mice. Cells from 1 patient were injected into 4 to 6 mice to reduce the individual differences.

\section{Measurement of Blood Flow and Clinical Score}

Blood flow in the ischemic hind limb was measured by using a laser Doppler perfusion imaging system (PeriScan PIM II; Lisca AB, Linkoping, Sweden) 28 days after treatment, as described previously. ${ }^{12}$ The recovery of perfusion in the ischemic hind limb of each mouse was estimated by the percentage of limb blood flow, which was calculated by the average perfusion in the left hind limb compared with that in the normal right hind limb. We also recorded the clinical scores of the ischemic hind limbs in all mice 28 days after treatment, as described previously. ${ }^{13}$ The mean blood flow and mean clinical scores of 4 to 6 mice represented the data of 1 patient and were used for statistical analysis.

\section{Statistical Analysis}

Data are expressed as means \pm SDs. Statistical significance between 2 groups was determined by using the 2-tailed unpaired $t$ test.

\section{RESULTS \\ Interindividual Differences of Angiogenic Potency of Bone Marrow Cells From Patients}

The bone marrow cells from different patients achieved different degrees of improvement in regional blood flow and the clinical score of the ischemic hind limbs of mice 28 days after cell implantation (Figure 1). Blood flow recovery and the clinical score of the ischemic limbs were better in mice receiving bone marrow cells from younger patients, but poor blood flow and clinical scores of ischemic limbs were observed in mice receiving bone marrow cells from older patients. The mean values of blood flow and clinical score of 4 to 6 mice receiving bone marrow cells from each patient were used for statistical analysis.

\section{Advanced Age Contributes to the Impairment of Angiogenic Potency of Bone Marrow Cells}

Compared with the young patients ( $<65$ years), bone marrow cells from patients with advanced age ( $\geq 65$ years) consisted of significantly less c-kit- and CD34-positive cells $(P<.01$; Figure $2, A)$. In culture, bone marrow cells from patients with advanced age produced less VEGF and formed fewer colonies than those from younger patients $(P<.01$; Figure $2, B)$. The blood flow and clinical scores were significantly worse in the ischemic limbs of mice that received bone marrow cells from patients with advanced age $(\geq 65$ years) than those from younger patients $(<65$ years; $P<.01$; Figure 2,C). Furthermore, the age of patients significantly and linearly correlated with both the blood flow $(r=-0.565, P=.003)$ and clinical scores $(r=0.432$, $P=.03)$ in the ischemic limbs of mice receiving bone marrow cells from the patients.

\section{Anemia Is a Risk Factor for Poor Angiogenic Potency of Bone Marrow Cells}

Compared with patients with normal hemoglobin values $(\geq 12 \mathrm{~g} / \mathrm{dL})$, bone marrow cells from patients with anemia (hemoglobin, $<12 \mathrm{~g} / \mathrm{dL}$ ) had significantly less c-kit- and CD34-positive stem cells $(P<.01$; Figure $3, A)$. In vitro analysis showed that bone marrow cells from patients with anemia produced less VEGF and formed fewer colonies $(P<.05$; Figure $3, B)$. The blood flow and clinical scores were significantly worse in the ischemic limbs of mice that received the implantation of bone marrow cells from patients with anemia than from those with normal hemoglobin values 

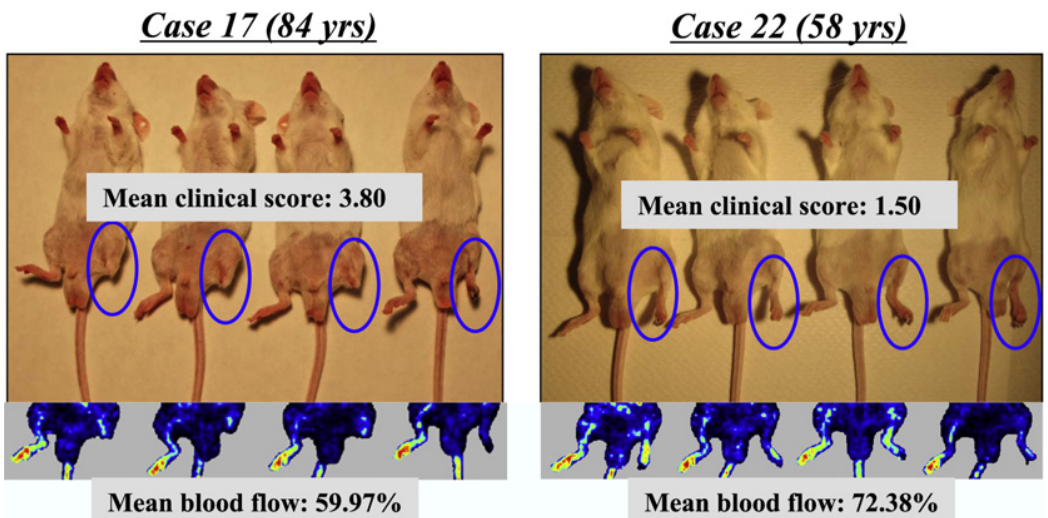

FIGURE 1. In vivo assessment of the angiogenic potency of bone marrow cells from patients using an ischemic limb model in SCID mice. Representative images show the improved appearance (top) and blood flow (below) in ischemic limbs of mice 28 days after the implantation of bone marrow cells from 2 patients. The clinical scores and blood flow were obviously worse in the mice implanted with bone marrow cells from the elderly patients (left) than in those implanted with bone marrow cells from the younger patients (right). Data are expressed as the means \pm SDs of 4 mice injected with bone marrow cells from 1 patient.

$(P<.05$; Figure 3,C). Furthermore, the hemoglobin levels of patients significantly and linearly correlated with the blood flow $(r=0.522, P=.007)$ and clinical scores $(r=-0.438, P=.03)$ in the ischemic limbs of mice receiving bone marrow cells from the patients.

\section{Poor Angiogenic Potency of Bone Marrow Cells From Patients With Renal Failure}

We found that bone marrow cells from patients with renal failure (dialysis) also had significantly less c-kit- and CD34positive stem cells than those from patients without renal failure $(P<.05$; Figure $4, A)$. Although the production of VEGF by bone marrow cells did not differ between the 2 groups, the number of colony-forming units seen on culturing bone marrow cells from patients with renal failure were significantly less than those from patients without renal failure $(P<.05$; Figure $4, B)$. Furthermore, the blood flow and clinical scores were also significantly worse in the ischemic limbs of mice that received bone marrow cells from patients with renal failure than those from patients without renal failure $(P<.05$; Figure $4, C)$.

\section{Diabetes and Hypertension Did Not Contribute to Decreased Angiogenic Potency of Bone Marrow Cells}

Unexpectedly, the data from our in vitro experiments showed that the number of c-kit- and CD34-positive stem cells in bone marrow did not decrease significantly in patients with diabetes (all are insulin independent; Figures 5, $A$, and $6, A$ ) when compared with numbers seen in their control cohorts. The in vitro production of VEGF by bone marrow cells also did not differ significantly between diabetic and nondiabetic patients, although the formation of colonies tended to decrease in the diabetic patients $(P=.07$; Figure 5, $B)$. No difference in the in vitro production of VEGF and colony formation from bone marrow cells was observed between patients with hypertension and patients without hypertension (Figure $6, B$ ). In vivo studies also showed that the blood flow and clinical scores were not different in the ischemic limbs of mice that received bone marrow cells from patients with diabetes and hypertension when compared with their control cohorts (Figures 5, C, and 6, C).

\section{DISCUSSION}

In this study we examined the quality and angiogenic potency of bone marrow cells from 25 patients with different backgrounds. We found that bone marrow cells from different patients had a different proportion of c-kit- and CD34positive stem cells, different degrees of VEGF production and colony formation in vitro, and different potency to improve the blood flow and clinical scores of ischemic limbs in mice after implantation. Furthermore, the angiogenic potency of bone marrow cells from patients was closely related to the number of c-kit- and CD34-positive stem cells in their bone marrow (data not shown). These data support that the angiogenic potency of bone marrow cells has obvious interindividual differences among patients.

We found that bone marrow cells from patients with advanced age had poor improvement in blood flow to the ischemic limbs of mice. Experimental studies have found that bone marrow cells from young but not old mice incorporated into the neovasculature and restored cardiac angiogenic functions. ${ }^{10,14} \mathrm{~A}$ reduced number of granulocyte-macrophage colony-forming units of the bone marrow cells from patients with advance age has also been reported. ${ }^{15}$ Our study has further demonstrated in vivo the age-related impairment of angiogenic potency of bone marrow cells from patients with advanced age. The molecular mechanisms for age-related functional impairment of bone marrow cells could be very complex, including cell senescence, enhanced oxidative stress, and others. ${ }^{16-18}$ It has been found that aging is 


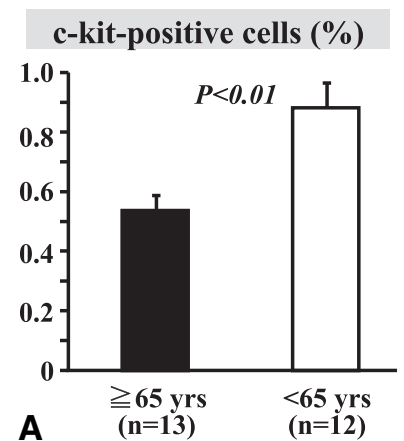

VEGF (ng/ml medium)
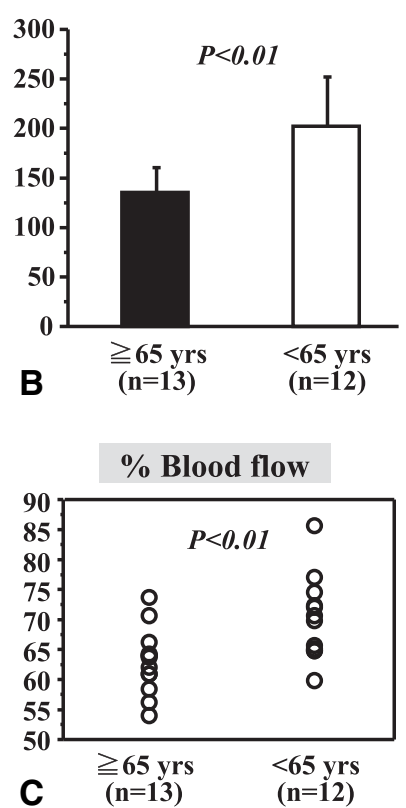
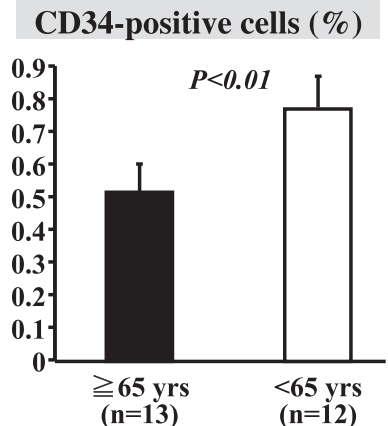

Colony forming units
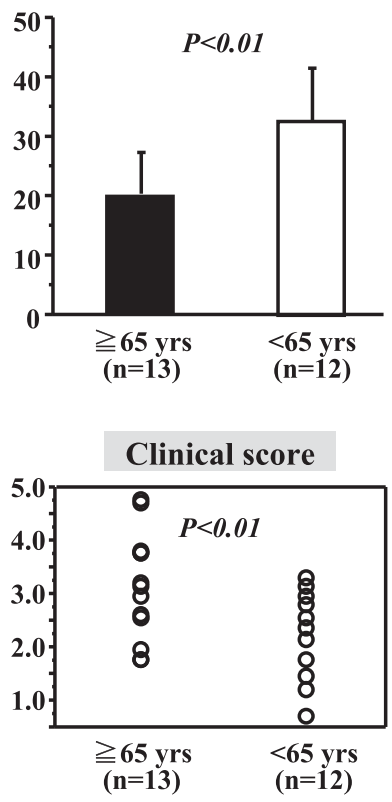

FIGURE 2. Comparison of the angiogenic potency of bone marrow cells from the elderly and young patients. A, The bone marrow cells from patients of advanced age had significantly lower numbers of c-kit-positive (left) and CD34-positive (right) stem cells than those from younger patients. B, The concentration of vascular endothelial growth factor $(V E G F)$ in the conditioned medium (left) and the number of colony-forming units (right) were significantly lower after 3 or 7 days of cultivation of bone marrow cells from the elderly patients than those from the young patients. C, The blood flow (left) and clinical scores (right) in the ischemic limbs of mice were significantly worse 28 days after implantation with bone marrow cells from the elderly patients than those from the young patients. Each circle represents the mean value of blood flow and clinical score of 4 to 6 mice injected with bone marrow cells of 1 patient.

associated with a reduction of telomere length in bone marrow cells, and the reduction of telomere length was associated with a reduced ex vivo migratory response. ${ }^{17}$ This indicates that age-associated telomere reduction might contribute to functional impairment of bone marrow cells. However, the precise mechanisms of age-related functional impairment in bone marrow cells were not completely understood, and we did not investigate the relative mechanisms in this study. c-kit-positive cells (\%)

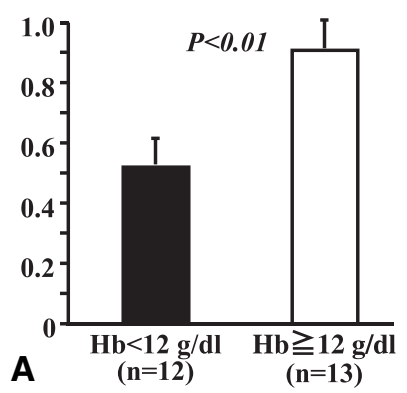

VEGF (ng/ml medium)
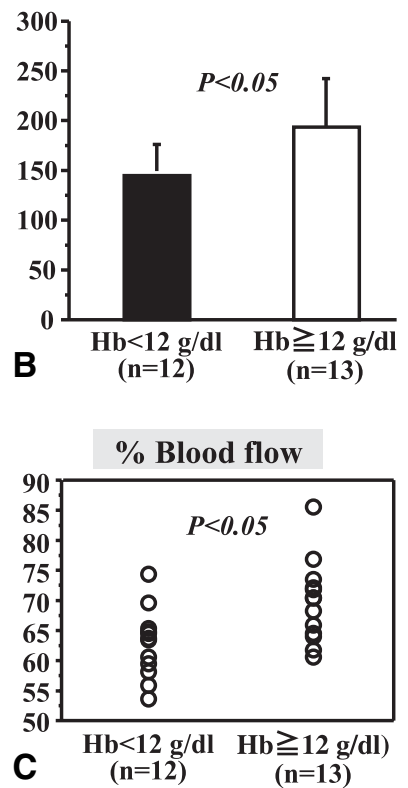

CD34-positive cells (\%)

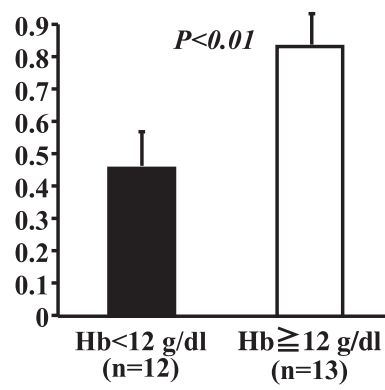

Colony forming units
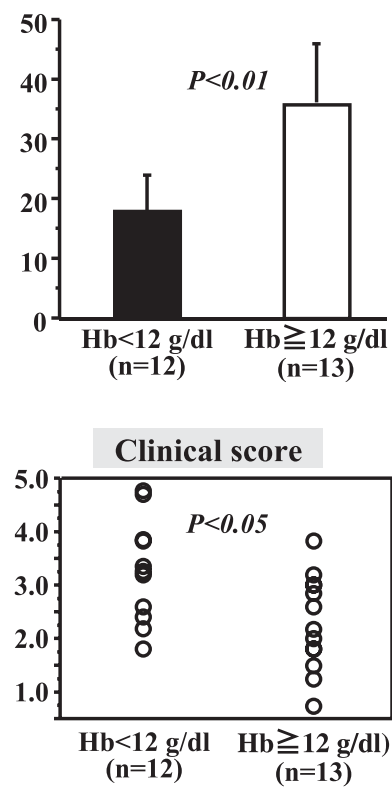

FIGURE 3. Angiogenic potency of bone marrow cells from patients with or without anemia. A, The bone marrow cells from patients with anemia (hemoglobin $[H b],<12 \mathrm{~g} / \mathrm{dL}$ ) had significantly lower numbers of c-kit-positive (left) and CD34-positive (right) stem cells than those from patients without anemia. B, The concentration of vascular endothelial growth factor (VEGF) in the conditioned medium (left) and the number of colony-forming units (right) were significantly lower after 3 or 7 days of cultivation of bone marrow cells from patients with anemia than from those without anemia. $\mathrm{C}$, The blood flow (left) and clinical scores ( right) in the ischemic limbs of mice were significantly worse 28 days after the implantation with bone marrow cells from patients with anemia than from those without. Each circle represents the mean value of blood flow and clinical scores of 4 to 6 mice injected with bone marrow cells of 1 patient.

We have also found that bone marrow cells from patients with renal failure had a deceased angiogenic potency in vivo, although a previous study has demonstrated impaired migratory activity and adhesion of endothelial progenitor cells in patients receiving long-term hemodialysis. ${ }^{19}$ Interestingly, a low hemoglobin level in the patients was found to relate significantly to the poor angiogenic potency of their bone marrow cells. Because these patients had no signs of acute 

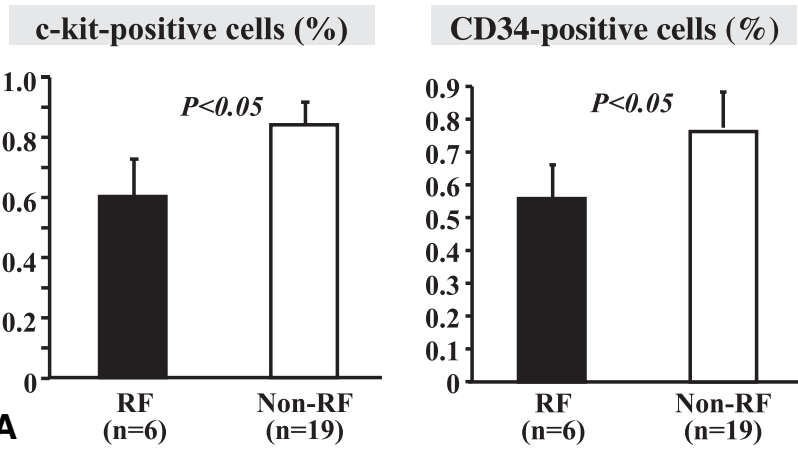

\section{VEGF (ng/ml medium)}
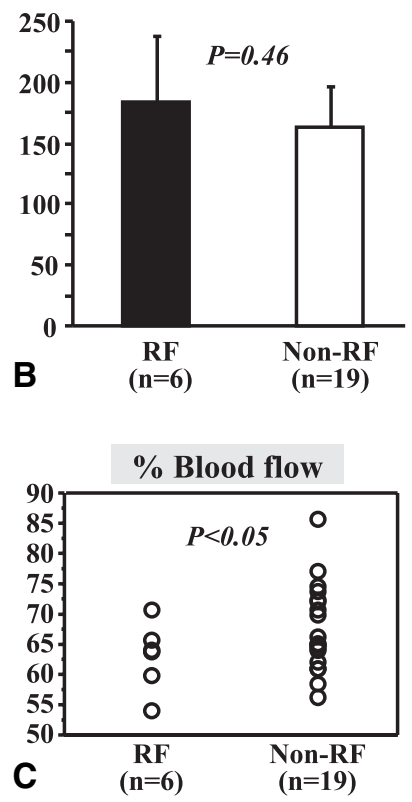

Colony forming units
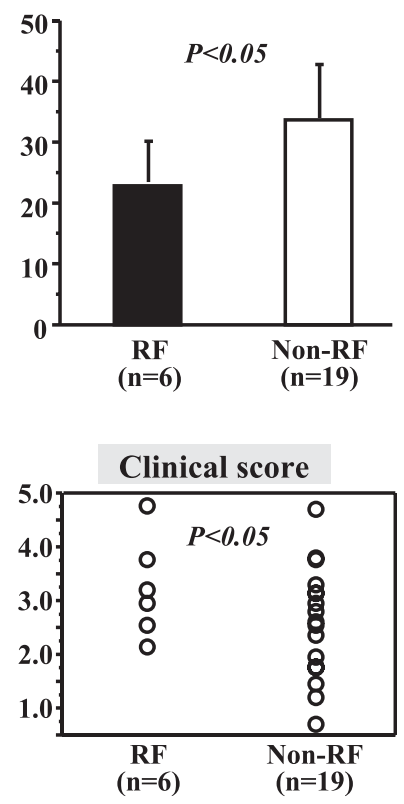

FIGURE 4. Angiogenic potency of bone marrow cells from patients with or without renal failure $(R F)$. A, The bone marrow cells from patients with renal failure (dialysis) had significantly lower numbers of c-kit-positive (left) and CD34-positive (right) stem cells than those from their control cohorts. B, The number of colony-forming units (right) were significantly lower after 7 days of cultivation of bone marrow cells from the patients with renal failure than from those without, but the concentration of vascular endothelial growth factor $(V E G F)$ in the conditioned medium (left) after 3 days of cultivation did not differ between groups. C, The blood flow (left) and clinical scores (right) in the ischemic limbs of mice were significantly worse 28 days after the implantation with bone marrow cells from patients with renal failure than from those without. Each circle represents the mean value of blood flow and clinical scores of 4 to 6 mice injected with bone marrow cells of 1 patient.

or chronic blood loss, a low hemoglobin level implicates impaired hematopoietic function, which means a poor quality of bone marrow stem cells. Although anemia was very often observed in patients undergoing dialysis, comparable hemoglobin levels were observed between patients with and without renal failure $(11.2 \pm 1.7 \mathrm{vs} 11.8 \pm 1.9 \mathrm{~g} / \mathrm{dL}, P=.975)$ in our study. Therefore we considered that both renal failure

c-kit-positive cells (\%)

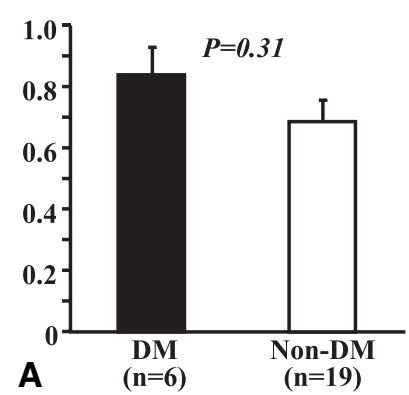

VEGF (ng/ml medium)

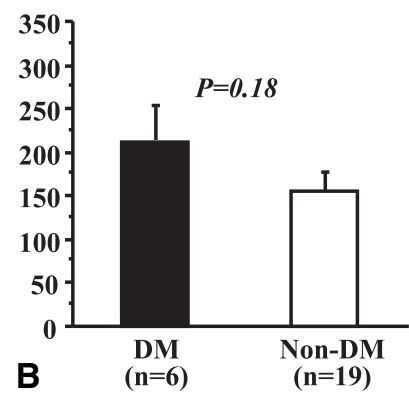

Colony forming units
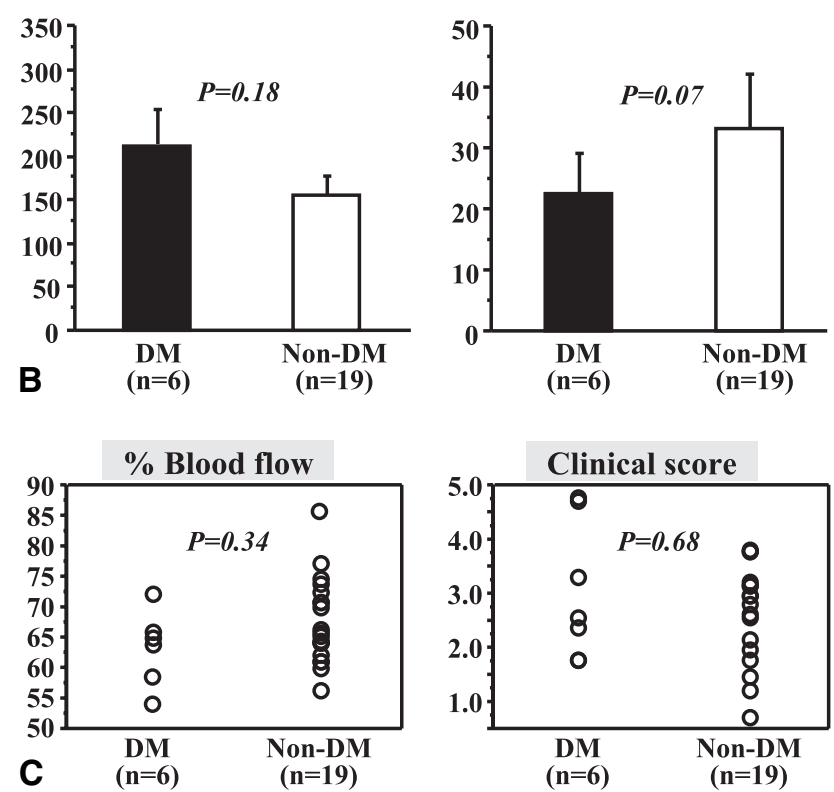

FIGURE 5. Comparison of the angiogenic potency of bone marrow cells from diabetic patients and their control cohorts. A, The numbers of c-kitpositive (left) and CD34-positive (right) stem cells in bone marrow did not differ between diabetic patients and their control cohorts. B, The concentration of vascular endothelial growth factor $(V E G F)$ in the conditioned medium (left) and the number of colony-forming units (right) were also not significantly different after 3 and 7 days of cultivation of bone marrow cells from diabetic patients and their control cohorts, although the number of colony-forming units tended to decrease in bone marrow cells from the diabetic patients. C, Bone marrow cells from diabetic and nondiabetic patients showed similar improvement of blood flow (left) and clinical scores (right) 28 days after implantation into the ischemic limbs of mice. Each circle represents the mean value of blood flow and clinical scores of 4 to 6 mice injected with bone marrow cells of 1 patient. DM, Diabetes mellitus.

and anemia are the risk factors related to poor angiogenic potency of bone marrow cells.

In contrast to previous investigations, ${ }^{7-9,18,20,21}$ we found that diabetes, hypertension, ischemic heart disease, and smoking (data not shown) were not risk factors for poor angiogenic potency of the bone marrow cells. These discrepancies might be attributed to the different cell sources and 
c-kit-positive cells (\%)
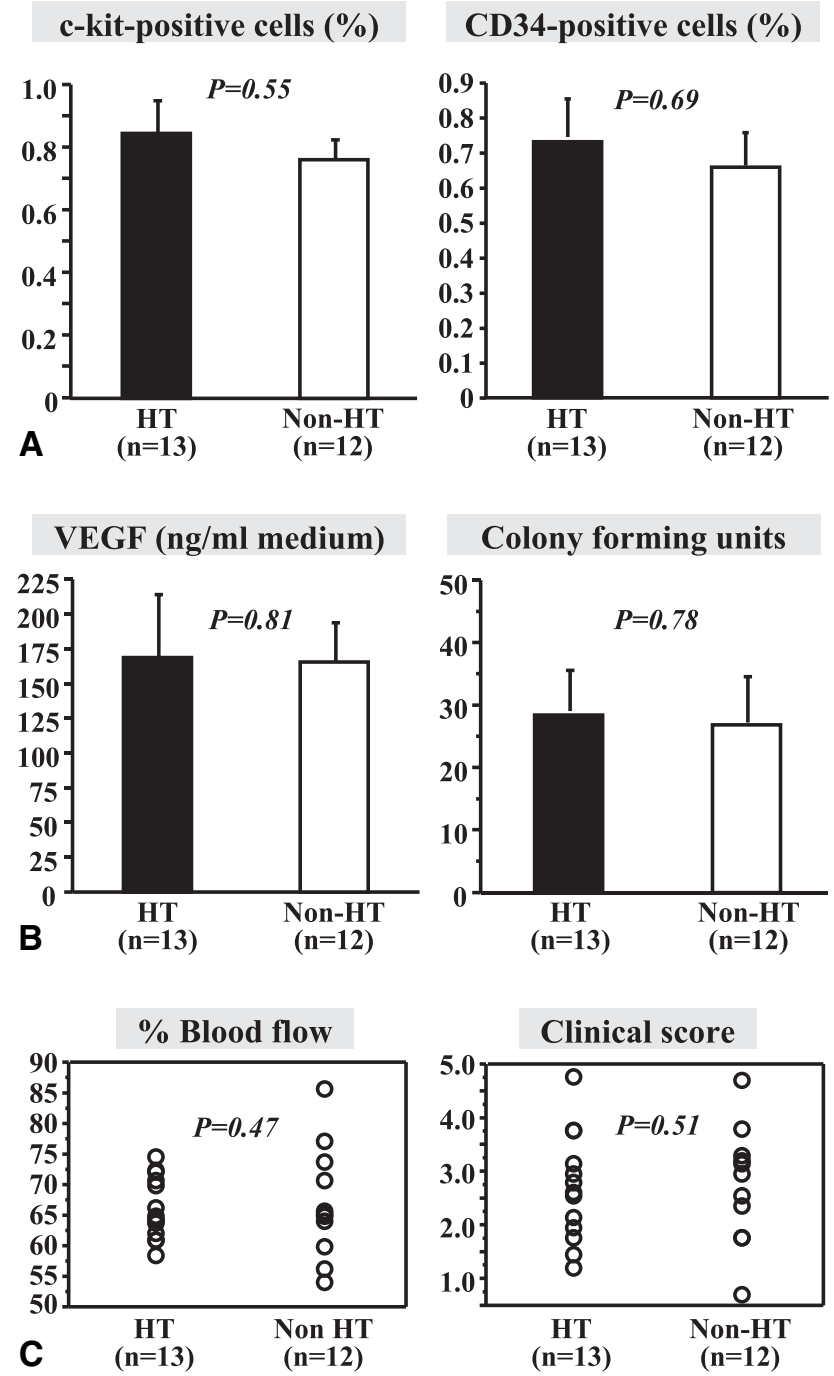

FIGURE 6. Comparison of the angiogenic potency of bone marrow cells from patients with or without hypertension $(H T)$. A, The numbers of ckit-positive (left) and CD34-positive (right) stem cells in bone marrow did not differ between patients with and without hypertension. B, The concentration of vascular endothelial growth factor $(V E G F)$ in the conditioned medium (left) and the number of colony-forming units (right) were also not significantly different after 3 and 7 days of cultivation of bone marrow cells from diabetic patients and their control cohorts, although the number of colony-forming units tended to decrease in bone marrow cells from the diabetic patients. C, Bone marrow cells from diabetic and nondiabetic patients showed similar improvement of blood flow (left) and clinical scores (right) 28 days after implantation into the ischemic limbs of mice. Each circle represents the mean value of blood flow and clinical scores of 4 to 6 mice injected with bone marrow cells of 1 patient.

experimental methods used among studies. The previous studies have used young healthy volunteers for the control group, ${ }^{21}$ but the control cohort in this study was composed of age-matched patients. Because advanced age was identified as one of the important risk factors contributing to functional impairment of bone marrow cells, it is not surprising that a worse function of bone marrow cells was found in these patients with ischemic heart disease (average age, 60 years) when compared with young healthy volunteers (average, 30 years) in the previous study. ${ }^{21}$

This study has several limitations. First, we examined the quality of bone marrow cells by culturing cells under normoxic conditions and estimated the angiogenic potency of the patients' bone marrow cells using an ischemic limb model of SCID mice. However, the ischemic tissue was specialized as a hypoxic microenviroment, and culturing cells under normoxic condition might not correctly reflect the potential functional difference of cells after implantation into the ischemic tissue. Otherwise, many other systemic and local angiogenic factors, including endothelial function, cytokines, and growth factors, differ among patients, and it would be impossible to reproduce these differences in our ischemic limb model in mice.

Second, the small sample size of this study might prevent us from detecting some potential risk factors (eg, diabetes) that have been demonstrated to contribute to the functional impairment of bone marrow cells.

Third, the small sample size of the study limited us to multivariate analysis. It is well know that renal failure, anemia, and old age are commonly associated in the same clinical process. Although the hemoglobin level is not different either between the aged and young patients or between the patients with and without renal failure, the independence of these risk factors need to be identified by means of further study of a large sample size with multivariate analysis.

Finally, we obtained bone marrow cells for study from about $15 \mathrm{~mL}$ of sternal marrow after anesthetic induction. However, many clinical trials collected cells by means of iliac aspiration of more $(50-500 \mathrm{~mL})$ bone marrow for implantation. The different methods might collect bone marrow cells with a different cell subpopulation and result in different angiogenic potency.

Although further studies with a large population size are required to identify and confirm our data, it is a well-accepted fact that functional impairment of autologous bone marrow cells can be induced by some systemic and regional disorders, and the implantation of autologous bone marrow cells in these patients might contribute to poor angiogenic potency. Because previous studies have found that the implantation of "young" cells into the aged mice induces effective angiogenesis, ${ }^{10,14}$ allografting of bone marrow cells from healthy donors could be an option for these patients with functional impairment of their bone marrow cells. Recent study has observed that allografting of mesenchymal stem cells into ischemic heart has functional benefit in sheep without an obvious immunologic problem. ${ }^{22}$ However, the immunologic and safety problems of allografting bone marrow mononuclear cells remain to be clarified because of the contamination of some lymphocytes in bone marrow mononuclear cells. Because the therapeutic potency of bone 
marrow-derived cells could be enhanced by gene modification, ${ }^{23}$ hypoxic preconditioning, ${ }^{24}$ and pretreatment with some drugs,${ }^{18}$ autologous bone marrow cells will be available for use in these patients if we can restore the impaired function of cells ex vivo before implantation.

In summary, we demonstrated that the quality and angiogenic potency of bone marrow cells obviously differs among patients with different clinical backgrounds. We identified that advanced age, renal failure, and anemia are risk factors related to poor angiogenic potency of bone marrow cells for the treatment of ischemic diseases.

\section{References}

1. Li TS, Murakami M, Kobayashi T, Shirasawa B, Mikamo A, Hamano K. Longterm efficacy and safety of the intramyocardial implantation of autologous bone marrow cells for the treatment of ischemic heart disease. $J$ Thorac Cardiovasc Surg. 2007;134:1347-9.

2. Wollert KC, Meyer GP, Lotz J, Ringes-Lichtenberg S, Lippolt P, Breidenbach C, et al. Intracoronary autologous bone-marrow cell transfer after myocardial infarction: the BOOST randomised controlled clinical trial. Lancet. 2004;364:141-8

3. Schächinger V, Erbs S, Elsässer A, Haberbosch W, Hambrecht R, Hölschermann $\mathrm{H}$, et al. Intracoronary bone marrow-derived progenitor cells in acute myocardial infarction. N Engl J Med. 2006;355:1210-21.

4. Lunde K, Solheim S, Aakhus S, Arnesen H, Abdelnoor M, Egeland T, et al. Intracoronary injection of mononuclear bone marrow cells in acute myocardial infarction. N Engl J Med. 2006;355:1199-209.

5. Seeger FH, Tonn T, Krzossok N, Zeiher AM, Dimmeler S. Cell isolation procedures matter: a comparison of different isolation protocols of bone marrow mononuclear cells used for cell therapy in patients with acute myocardial infarction. Eur Heart J. 2007;28:766-72.

6. Qin SL, Li TS, Kubo M, Ohshima M, Furutani A, Hamano K. Transient increase of cytokines in the acute ischemic tissue is beneficial to cell-based therapeutic angiogenesis. Circ J. 2008;72:2075-80.

7. Walter DH, Haendeler J, Reinhold J, Rochwalsky U, Seeger F, Honold J, et al. Impaired CXCR4 signaling contributes to the reduced neovascularization capacity of endothelial progenitor cells from patients with coronary artery disease. Circ Res. 2005;97:1142-51.

8. Li TS, Furutani A, Takahashi M, Ohshima M, Qin SL, Kobayashi T, et al. Impaired potency of bone marrow mononuclear cells for inducing therapeutic angiogenesis in obese diabetic rats. Am J Physiol Heart Circ Physiol. 2006;290: H1362-9.

9. Tamarat R, Silvestre JS, Le Ricousse-Roussanne S, Barateau V, LecomteRaclet L, Clergue M, et al. Impairment in ischemia-induced neovascularization in diabetes: bone marrow mononuclear cell dysfunction and therapeutic potential of placenta growth factor treatment. Am J Pathol. 2004;164:57-66.
10. Zhang H, Fazel S, Tian H, Mickle DA, Weisel RD, Fujii T, et al. Increasing donor age adversely impacts beneficial effects of bone marrow but not smooth muscle myocardial cell therapy. Am J Physiol Heart Circ Physiol. 2005;289: H2089-6.

11. Li TS, Suzuki R, Ueda K, Murata T, Hamano K. Analysis of the origin and population dynamics of cardiac progenitor cells in a donor heart model. Stem Cells. 2007;25:911-7.

12. Li TS, Ito H, Hayashi M, Furutani A, Matsuzaki M, Hamano K. Cellular expression of integrin-beta 1 is of critical importance for inducing therapeutic angiogenesis by cell implantation. Cardiovasc Res. 2005;65:64-72.

13. Yu J, deMuinck ED, Zhuang Z, Drinane M, Kauser K, Rubanyi GM, et al. Endothelial nitric oxide synthase is critical for ischemic remodeling, mural cell recruitment, and blood flow reserve. Proc Natl Acad Sci U S A. 2005;102:10999-1004

14. Edelberg JM, Tang L, Hattori K, Lyden D, Rafii S. Young adult bone marrowderived endothelial precursor cells restore aging-impaired cardiac angiogenic function. Circ Res. 2002;90:e89-93.

15. Kissel CK, Lehmann R, Assmus B, Aicher A, Honold J, Fischer-Rasokat U, et al. Selective functional exhaustion of hematopoietic progenitor cells in the bone marrow of patients with postinfarction heart failure. J Am Coll Cardiol. 2007;49:2341-9.

16. Dimmeler S, Leri A. Aging and disease as modifiers of efficacy of cell therapy. Circ Res. 2008;102:1319-30.

17. Spyridopoulos I, Erben Y, Brummendorf TH, Haendeler J, Dietz K, Seeger F, et al. Telomere gap between granulocytes and lymphocytes is a determinant for haematopoetic progenitor cell impairment in patients with previous myocardial infarction. Arterioscler Thromb Vasc Biol. 2008;28:968-74.

18. Sorrentino SA, Bahlmann FH, Besler C, Müller M, Schulz S, Kirchhoff N, et al Oxidant stress impairs in vivo reendothelialization capacity of endothelial progenitor cells from patients with type 2 diabetes mellitus: restoration by the peroxisome proliferator-activated receptor-gamma agonist rosiglitazone. Circulation. 2007; 116:163-73.

19. Herbrig K, Pistrosch F, Oelschlaegel U, Wichmann G, Wagner A, Foerster S, et al Increased total number but impaired migratory activity and adhesion of endothelial progenitor cells in patients on long-term hemodialysis. Am J Kidney Dis. 2004; 44:840-9.

20. Kondo T, Hayashi M, Takeshita K, Numaguchi Y, Kobayashi K, Iino S, et al. Smoking cessation rapidly increases circulating progenitor cells in peripheral blood in chronic smokers. Arterioscler Thromb Vasc Biol. 2004;24:1442-7.

21. Heeschen C, Lehmann R, Honold J, Assmus B, Aicher A, Walter DH, et al. Profoundly reduced neovascularization capacity of bone marrow mononuclear cells derived from patients with chronic ischemic heart disease. Circulation. 2004; 109:1615-22.

22. Hamamoto H, Gorman JH 3rd, Ryan LP, Hinmon R, Martens TP, Schuster MD, et al. Allogeneic mesenchymal precursor cell therapy to limit remodeling after myocardial infarction: the effect of cell dosage. Ann Thorac Surg. 2009;87: 794-801.

23. Mangi AA, Noiseux N, Kong D, He H, Rezvani M, Ingwall JS, et al. Mesenchymal stem cells modified with Akt prevent remodeling and restore performance of infarcted hearts. Nat Med. 2003;9:1195-201.

24. Li TS, Hamano K, Suzuki K, Ito H, Zempo N, Matsuzaki M. Improved angiogenic potency by implantation of ex vivo hypoxia prestimulated bone marrow cells in rats. Am J Physiol Heart Circ Physiol. 2002;283:H468-73. 